PROCEEDINGS OF THE

AMERICAN MATHEMATICAL SOCIETY

Volume 138, Number 7, July 2010, Pages 2513-2519

S 0002-9939(10)10301-3

Article electronically published on March 4, 2010

\title{
UNIFORM ASYMPTOTIC EXPANSIONS OF THE TRICOMI-CARLITZ POLYNOMIALS
}

\author{
K. F. LEE AND R. WONG
}

(Communicated by Walter Van Assche)

Dedicated to the Lord

\begin{abstract}
The Tricomi-Carlitz polynomials satisfy the second-order linear difference equation

$$
(n+1) f_{n+1}^{(\alpha)}(x)-(n+\alpha) x f_{n}^{(\alpha)}(x)+f_{n-1}^{(\alpha)}(x)=0, \quad n \geq 1,
$$

with initial values $f_{0}^{(\alpha)}(x)=1$ and $f_{1}^{(\alpha)}(x)=\alpha x$, where $x$ is a real variable and $\alpha$ is a positive parameter. An asymptotic expansion is derived for these polynomials by using the turning-point theory for three-term recurrence relations developed by Wang and Wong [Numer. Math. 91(2002) and 94(2003)]. The result holds uniformly in regions containing the critical values $x= \pm 2 / \sqrt{\nu}$, where $\nu=n+2 \alpha-1 / 2$.
\end{abstract}

\section{INTRODUCTION}

The Tricomi polynomials are defined by

$$
t_{n}^{(\alpha)}(x)=\sum_{k=0}^{n}(-1)^{k}\left(\begin{array}{c}
x-\alpha \\
k
\end{array}\right) \frac{x^{n-k}}{(n-k) !}, \quad n=0,1,2, \ldots,
$$

which satisfy the recurrence relation

$$
(n+1) t_{n+1}^{(\alpha)}(x)-(n+\alpha) t_{n}^{(\alpha)}(x)+x t_{n-1}^{(\alpha)}(x)=0, \quad n \geq 1,
$$

with initial values $t_{0}^{(\alpha)}(x)=1, t_{1}^{(\alpha)}(x)=\alpha$. Tricomi [7] observed that $\left\{t_{n}^{(\alpha)}(x)\right\}$ is not a system of orthogonal polynomials, since the recurrence relation (1.2) fails to have the required form [6, p.135]. However, Carlitz [1 discovered that if one sets

$$
f_{n}^{(\alpha)}(x)=x^{n} t_{n}^{(\alpha)}\left(x^{-2}\right),
$$

then $f_{n}^{(\alpha)}(x)$ satisfies

$$
(n+1) f_{n+1}^{(\alpha)}(x)-(n+\alpha) x f_{n}^{(\alpha)}(x)+f_{n-1}^{(\alpha)}(x)=0, \quad n \geq 1,
$$

Received by the editors June 23, 2009, and, in revised form, November 6, 2009. 2010 Mathematics Subject Classification. Primary 41A60, 39A10; Secondary 33C45.

Key words and phrases. Tricomi-Carlitz polynomials, uniform asymptotic expansion, difference equation.

(C)2010 American Mathematical Society Reverts to public domain 28 years from publication 
with initial values $f_{0}^{(\alpha)}(x)=1, f_{1}^{(\alpha)}(x)=\alpha x$. Furthermore, he gave the orthogonal relation

$$
\int_{-\infty}^{\infty} f_{m}^{(\alpha)}(x) f_{n}^{(\alpha)}(x) d \psi^{(\alpha)}(x)=\frac{2 e^{\alpha}}{(n+\alpha) n !} \delta_{m n},
$$

where $\psi^{(\alpha)}(x)$ is the step function whose jumps are

$$
d \psi^{(\alpha)}(x)=\frac{(k+\alpha)^{k-1} e^{-k}}{k !} \text { at } \quad x=x_{k}= \pm(k+\alpha)^{-1 / 2}, \quad k=0,1,2, \ldots
$$

The generating function

$$
\exp \left\{\frac{w}{x}+\frac{1-\alpha x^{2}}{x^{2}} \log (1-w x)\right\}=\sum_{n=0}^{\infty} f_{n}^{(\alpha)}(x) w^{n}
$$

can be derived from the recurrence relation (1.4) when $x \neq 0$. The series in (1.7) is convergent for $|w x|<1$.

Asymptotic behavior of $f_{n}^{(\alpha)}(x)$ has been investigated by Goh and Wimp [2, 3]. In their first paper, they used an elementary approach to show that

$$
\frac{f_{n}^{(\alpha)}(y / \sqrt{\alpha})}{\alpha^{-n / 2} y^{n} n^{-\alpha\left(1-y^{2}\right) / y^{2}-1}} \rightarrow \frac{e^{\alpha / y^{2}}}{\Gamma\left(-\alpha\left(1-y^{2}\right) / y^{2}\right)}, \quad \text { as } n \rightarrow \infty,
$$

uniformly for all $y$ in a compact set $K \subseteq \mathbb{C} \backslash[-1,1]$. (There is a minor error in the statement of their result; the factor $\alpha^{-n / 2}$ in the denominator on the left-hand side of (1.8) is missing in their equation (23) in 2.) The validity of (1.8) can be verified by a direct application of Darboux's method [10, p. 116]. Goh and Wimp also observed that all zeros of $f_{n}^{(\alpha)}(y / \sqrt{\alpha})$ lie in the interval $[-1,1]$. In their second paper, they used a saddle-point method to study the asymptotics of $f_{n}^{(\alpha)}(z / \sqrt{n})$ for $z$ in neighborhoods of $z= \pm i / 2$. Note that the scales in their two papers are different; in 22 the scale is $y / \sqrt{\alpha}$, whereas in 3 the scale is $z / \sqrt{n}$. The behavior of $f_{n}^{(\alpha)}(x)$ as $\alpha \rightarrow \infty$ has been studied by López and Temme 4 . Their result is expressed in terms of Hermite polynomials.

The purpose of this paper is to present an asymptotic expansion for $f_{n}^{(\alpha)}(t / \sqrt{\nu})$, which holds uniformly for $t$ in $[0, \infty)$, where $\nu=n+2 \alpha-1 / 2$. In view of the reflection formula $f_{n}^{(\alpha)}(-x)=(-1)^{n} f_{n}^{(\alpha)}(x)$, a corresponding result can be given for $t$ in $(-\infty, 0]$. Note that our result is not covered in the two papers of Goh and Wimp; in fact, it complements those in [2] and 3]. We also point out that the Tricomi-Carlitz polynomials do not satisfy a second-order differential equation; hence the powerful tools developed for differential equations (see, e.g., 5]) are not applicable. Our approach is to use a turning-point theory recently introduced by Wang and Wong [8, 9] for three-term recurrence relations.

\section{Difference Equation}

Returning to (1.4), we write

$$
f_{n+1}^{(\alpha)}(x)-\frac{n+\alpha}{n+1} x f_{n}^{(\alpha)}(x)+\frac{1}{n+1} f_{n-1}^{(\alpha)}(x)=0
$$

and introduce the sequence $\left\{K_{n}\right\}$ defined by

$$
(n+1) K_{n+1}=K_{n-1}
$$


with $K_{0}=1$ and $K_{1}=\sqrt{2 / \pi}$. Induction shows that

$$
K_{n}=\frac{1}{2^{n / 2} \Gamma\left(\frac{1}{2} n+1\right)} .
$$

With the notation

$$
F_{n}^{(\alpha)}(x):=\frac{f_{n}^{(\alpha)}(x)}{K_{n}}
$$

equation (2.1) can be put in the canonical form considered in [9,

$$
F_{n+1}^{(\alpha)}(x)-\left(A_{n} x+B_{n}\right) F_{n}^{(\alpha)}(x)+F_{n-1}^{(\alpha)}(x)=0
$$

with

$$
A_{n}=\frac{n+\alpha}{n+1} \frac{K_{n}}{K_{n+1}}=\frac{n+\alpha}{\sqrt{2}} \frac{\Gamma\left(\frac{1}{2}(n+1)\right)}{\Gamma\left(\frac{1}{2} n+1\right)}
$$

and $B_{n}=0$. To find an asymptotic expansion for $A_{n}$, we recall the well-known result [10, p. 47]

$$
\frac{\Gamma\left(\frac{1}{2} n+\frac{1}{2}\right)}{\Gamma\left(\frac{1}{2} n+1\right)} \sim \sqrt{\frac{2}{n}}\left[1-\frac{1}{4 n}+\frac{1}{32 n^{2}}+\cdots\right] .
$$

Thus,

$$
A_{n} \sim n^{1 / 2}\left[1+\left(\alpha-\frac{1}{4}\right) \frac{1}{n}+\left(\frac{1}{32}-\frac{\alpha}{4}\right) \frac{1}{n^{2}}+\cdots\right] .
$$

In terms of the notation

$$
A_{n} \sim n^{-\theta} \sum_{s=0}^{\infty} \frac{\alpha_{s}}{n^{s}} \quad \text { and } \quad B_{n} \sim \sum_{s=0}^{\infty} \frac{\beta_{s}}{n^{s}},
$$

used in [9], we have

$$
\theta=-\frac{1}{2}, \quad \alpha_{0}=1, \quad \alpha_{1}=\alpha-\frac{1}{4}, \quad \alpha_{2}=\left(\frac{1}{32}-\frac{\alpha}{4}\right), \cdots
$$

and $\beta_{0}=\beta_{1}=\beta_{2}=\cdots=0$. If these expansions are recast in the form

$$
A_{n} \sim \nu^{-\theta} \sum_{s=0}^{\infty} \frac{\alpha_{s}^{\prime}}{\nu^{s}} \quad \text { and } \quad B_{n} \sim \sum_{s=0}^{\infty} \frac{\beta_{s}^{\prime}}{\nu^{s}},
$$

where $\nu=n+\tau_{0}$ and $\tau_{0}$ is some fixed real number to be determined, it is easily found that

$$
\alpha_{0}^{\prime}=1, \quad \alpha_{1}^{\prime}=\left(\alpha-\frac{1}{4}\right)-\frac{\tau_{0}}{2}, \cdots
$$

and $\beta_{0}^{\prime}=\beta_{1}^{\prime}=\beta_{2}^{\prime}=\cdots=0$. To apply the result in [9], we first choose $\tau_{0}$ so that $\alpha_{1}^{\prime}=0$. From (2.11), it is obvious that the choice is

$$
\tau_{0}=2 \alpha-\frac{1}{2} .
$$

According to equation (2.4) in [9], the characteristic equation is

$$
\lambda^{2}-t \lambda+1=0,
$$

where $t$ is the rescaled variable $x=\nu^{-\frac{1}{2}} t$. The two roots of this equation are

$$
\lambda_{ \pm}=\frac{1}{2}\left(t \pm \sqrt{t^{2}-4}\right) .
$$


The points $t_{ \pm}= \pm 2$ where the two roots coincide are called the turning points of equation (2.5). In view of the symmetry relation $F_{n}^{(\alpha)}(-x)=(-1)^{n} F_{n}^{(\alpha)}(x)$, we may restrict ourselves just to the case $0<t<\infty$.

We now define the function $\zeta(t)$ introduced in [9, (4.10)]. With $t_{+}=2, \theta=-\frac{1}{2}$, $\alpha_{0}^{\prime}=1$ and $\beta_{0}^{\prime}=0$, this function is given by

$$
\frac{2}{3}[\zeta(t)]^{3 / 2}:=\log \frac{t+\sqrt{t^{2}-4}}{2}-\frac{1}{t^{2}} \int_{2}^{t} \frac{s^{2}}{\sqrt{s^{2}-4}} d s, \quad t \geq 2,
$$

and

$$
\frac{2}{3}[-\zeta(t)]^{3 / 2}:=t^{-2} \int_{t}^{2} \frac{s^{2}}{\sqrt{4-s^{2}}} d s-\cos ^{-1} \frac{t}{2}, \quad 0<t<2 .
$$

By direct calculation, one obtains

$$
\frac{2}{3}[\zeta(t)]^{3 / 2}=\log \frac{t+\sqrt{t^{2}-4}}{2}-\frac{1}{t^{2}}\left[\frac{1}{2} t \sqrt{t^{2}-4}+2 \log \left|t+\sqrt{t^{2}-4}\right|-2 \ln 2\right],
$$

for $t \geq 2$, and

$$
\frac{2}{3}[-\zeta(t)]^{3 / 2}=\frac{1}{t^{2}}\left[-2 \sin ^{-1} \frac{t}{2}+\pi+\frac{t}{2} \sqrt{4-t^{2}}\right]-\cos ^{-1} \frac{t}{2},
$$

for $0<t<2$. We also define the functions $H_{0}(\zeta)$ and $\Phi(\zeta)$ introduced in [9. (4.19) and (4.28)]. In the present situation, these functions are given by

$$
H_{0}(\zeta)=-\sqrt{\frac{t^{2}-4}{4 \zeta}} \quad \text { and } \quad \Phi(\zeta)=0
$$

where $\zeta$ is the function defined in (2.15) and (2.16). Note that in our special case, $\alpha_{1}^{\prime}=\beta_{1}^{\prime}=0$; hence, according to the definition of $\Phi(\zeta)$ given in (4.28) of [9], the second equation in (2.17) holds for $0<t<\infty$, instead of $t \geq \delta, 0<\delta<2$.

With this preliminary work done, we can now apply the main result in [9] to conclude that there are constants $C_{1}(x)$ and $C_{2}(x)$ such that the polynomials $F_{n}^{(\alpha)}(x)$ in (2.4) can be expressed as

$$
F_{n}^{(\alpha)}(x)=C_{1}(x) P_{n}(x)+C_{2}(x) Q_{n}(x),
$$

where, with $x=\nu^{-\frac{1}{2}} t, P_{n}(x)$ and $Q_{n}(x)$ have the asymptotic expansions

$$
P_{n}\left(\nu^{-\frac{1}{2}} t\right) \sim\left(\frac{4 \zeta}{t^{2}-4}\right)^{\frac{1}{4}}\left[\operatorname{Ai}\left(\nu^{\frac{2}{3}} \zeta\right) \sum_{s=0}^{\infty} \frac{\widetilde{A}_{s}(\zeta)}{\nu^{s-\frac{1}{6}}}+\mathrm{Ai}^{\prime}\left(\nu^{\frac{2}{3}} \zeta\right) \sum_{s=0}^{\infty} \frac{\widetilde{B}_{s}(\zeta)}{\nu^{s+\frac{1}{6}}}\right]
$$

and

$$
Q_{n}\left(\nu^{-\frac{1}{2}} t\right) \sim\left(\frac{4 \zeta}{t^{2}-4}\right)^{\frac{1}{4}}\left[\operatorname{Bi}\left(\nu^{\frac{2}{3}} \zeta\right) \sum_{s=0}^{\infty} \frac{\widetilde{A}_{s}(\zeta)}{\nu^{s-\frac{1}{6}}}+\operatorname{Bi}^{\prime}\left(\nu^{\frac{2}{3}} \zeta\right) \sum_{s=0}^{\infty} \frac{\widetilde{B}_{s}(\zeta)}{\nu^{s+\frac{1}{6}}}\right] .
$$

In (2.19) and (2.20), $\mathrm{Ai}(\cdot)$ and $\operatorname{Bi}(\cdot)$ are the Airy functions, the leading coefficients are given by

$$
\widetilde{A}_{0}(\zeta)=1 \quad \text { and } \quad \widetilde{B}_{0}(\zeta)=0,
$$

and the expansions hold uniformly for $0 \leq t<\infty$. 


\section{Determination of $C_{1}(x)$ and $C_{2}(x)$}

First we examine the behavior of $f_{n}^{(\alpha)}(x)$ as $n \rightarrow \infty$. By using the classical steepest decent method, one can show that to leading order, we have

$$
\begin{aligned}
f_{n}^{(\alpha)}\left(\frac{t}{\sqrt{\nu}}\right) \sim & \frac{\nu^{-\frac{n}{2}} e^{\nu\left(\frac{\lambda_{+}}{t}+\left(\frac{2}{t^{2}}-1\right) \log \lambda_{+}\right)}}{\sqrt{2 \pi \nu}\left(t^{2}-4\right)^{\frac{1}{4}}} \cos \left(\pi \alpha-\frac{\nu \pi}{t^{2}}\right) \\
& +\frac{\nu^{-\frac{n}{2}} e^{\nu\left(\frac{\lambda_{-}}{t}+\left(\frac{2}{t^{2}}-1\right) \log \lambda_{-}\right)}}{\sqrt{2 \pi \nu}\left(t^{2}-4\right)^{\frac{1}{4}}} 2 \sin \left(\pi \alpha-\frac{\nu \pi}{t^{2}}\right),
\end{aligned}
$$

when $t>2$;

$$
\begin{aligned}
f_{n}^{(\alpha)}\left(\frac{t}{\sqrt{\nu}}\right) \sim \frac{\sqrt{2} \nu^{-\frac{n}{2}} e^{\frac{\nu}{2}}}{\sqrt{\pi \nu}\left(4-t^{2}\right)^{\frac{1}{4}}} \sin [ & \nu\left(\frac{1}{2 t} \sqrt{4-t^{2}}+\frac{\pi}{t^{2}}-\frac{2}{t^{2}} \sin ^{-1} \frac{t}{2}-\cos ^{-1} \frac{t}{2}\right) \\
& \left.+\pi \alpha-\frac{\nu \pi}{t^{2}}+\frac{\pi}{4}\right],
\end{aligned}
$$

when $0<t<2$ and

$$
f_{n}^{(\alpha)}\left(\frac{2}{\sqrt{\nu}}\right) \sim \nu^{-\frac{n}{2}} e^{\frac{\nu}{2}} 3^{\frac{1}{3}} \nu^{-\frac{1}{3}}\left(\frac{1}{3 \Gamma\left(\frac{2}{3}\right)} \cos \left(\pi \alpha-\frac{\nu \pi}{4}\right)+\frac{\sqrt{3}}{3 \Gamma\left(\frac{2}{3}\right)} \sin \left(\pi \alpha-\frac{\nu \pi}{4}\right)\right),
$$

when $t=2$. Next we recall the well-known asymptotic formulas

$$
\operatorname{Ai}(\eta) \sim \frac{\eta^{-\frac{1}{4}}}{2 \sqrt{\pi}} \exp \left(-\frac{2}{3} \eta^{\frac{3}{2}}\right)
$$

and

$$
\operatorname{Bi}(\eta) \sim \frac{\eta^{-\frac{1}{4}}}{\sqrt{\pi}} \exp \left(\frac{2}{3} \eta^{\frac{3}{2}}\right)
$$

as $\eta \rightarrow+\infty$; see [5. p. 392]. Furthermore, since $\nu=n+2 \alpha-1 / 2$, it is readily seen from (3.4) and (3.5) that

$$
\begin{aligned}
& \frac{P_{n}\left(\nu^{-\frac{1}{2}} t\right)}{2^{\frac{n}{2}} \Gamma\left(\frac{n}{2}+1\right)} \sim \frac{\nu^{-\frac{n}{2}} \nu^{-\frac{1}{2}}}{\sqrt{2} \pi\left(t^{2}-4\right)^{\frac{1}{4}}} e^{\nu\left(\frac{\lambda_{+}}{t}+\left(\frac{2}{t^{2}}-1\right) \log \lambda_{+}\right),} \\
& \frac{Q_{n}\left(\nu^{-\frac{1}{2}} t\right)}{2^{\frac{n}{2}} \Gamma\left(\frac{n}{2}+1\right)} \sim \frac{\sqrt{2} \nu^{-\frac{n}{2}} \nu^{-\frac{1}{2}}}{\pi\left(t^{2}-4\right)^{\frac{1}{4}}} e^{\nu\left(\frac{\lambda_{-}}{t}+\left(\frac{2}{t^{2}}-1\right) \log \lambda_{-}\right)} .
\end{aligned}
$$

Comparing the two sides of (2.18), one concludes that

$$
C_{1}(x)=\sqrt{\pi} \cos \left(\pi \alpha-\frac{\pi}{x^{2}}\right), \quad C_{2}(x)=\sqrt{\pi} \sin \left(\pi \alpha-\frac{\pi}{x^{2}}\right) .
$$

Note that in obtaining (3.6), any one of the three formulas (3.1), (3.2) or (3.3) could have been used. In summary, we have from (2.4), (2.18), (2.19) and (2.20)

$$
\begin{aligned}
f_{n}^{(\alpha)}\left(\frac{t}{\sqrt{\nu}}\right)= & \frac{\sqrt{\pi} \cos \left(\pi \alpha-\frac{\nu \pi}{t^{2}}\right)}{2^{\frac{1}{2} n} \Gamma\left(\frac{1}{2} n+1\right)}\left(\frac{4 \zeta}{t^{2}-4}\right)^{\frac{1}{4}} \nu^{\frac{1}{6}}\left[\operatorname{Ai}\left(\nu^{\frac{2}{3}} \zeta\right)+O\left(\frac{1}{\nu}\right)\right] \\
& +\frac{\sqrt{\pi} \sin \left(\pi \alpha-\frac{\nu \pi}{t^{2}}\right)}{2^{\frac{1}{2} n} \Gamma\left(\frac{1}{2} n+1\right)}\left(\frac{4 \zeta}{t^{2}-4}\right)^{\frac{1}{4}} \nu^{\frac{1}{6}}\left[\operatorname{Bi}\left(\nu^{\frac{2}{3}} \zeta\right)+O\left(\frac{1}{\nu}\right)\right],
\end{aligned}
$$


where $x=\nu^{-\frac{1}{2}} t$ and $\nu=n+2 \alpha-1 / 2$. This result holds uniformly for $0 \leq t<\infty$. As a check on the validity of (3.7), we note that

$$
f_{2 n}^{(\alpha)}(0)=\frac{(-1)^{n}}{2^{n} n !}, \quad f_{2 n+1}^{(\alpha)}(0)=0, \quad n=0,1,2, \ldots
$$

From (3.7), we obtain

$$
f_{n}^{(\alpha)}(0) \sim \frac{e^{n / 2}}{\sqrt{\pi} n^{(n+1) / 2}} \sin \left(-\frac{n \pi}{2}+\frac{\pi}{2}\right) .
$$

In view of Stirling's formula, (3.9) agrees with (3.8).

\section{Numerical RESUlts}

The expansion (3.7) is particularly useful near $t=2$, where the two characteristic roots in (2.14) coincide. As an illustration, we take $\alpha=1.9$ and $n=100$. Table 1 provides exact and approximate values of $2^{n / 2} \Gamma(n / 2+1) f_{n}^{(\alpha)}(t / \sqrt{\nu})$. The last column of the table shows the percentage error of the approximations.

TABLE 1. Numerical Results

\begin{tabular}{cccc}
\hline $\mathrm{t}$ & \multicolumn{1}{c}{ Exact values } & Approximate values & Error \\
\hline 1.0 & $-7.46841313 \times 10^{-1}$ & $-7.37169075 \times 10^{-1}$ & 0.0130 \\
1.2 & $6.30560302 \times 10^{-1}$ & $6.34644034 \times 10^{-1}$ & 0.0065 \\
1.4 & $8.46491439 \times 10^{-1}$ & $8.31094179 \times 10^{-1}$ & 0.0182 \\
1.6 & -1.30620957 & -1.29091632 & 0.0117 \\
1.8 & 1.51877275 & 1.49426911 & 0.0161 \\
2.0 & 1.90931070 & 1.89864389 & 0.0056 \\
2.2 & $3.26791053 \times 10^{2}$ & $3.16621347 \times 10^{2}$ & 0.0311 \\
2.4 & $-9.21372780 \times 10^{4}$ & $-8.83780892 \times 10^{4}$ & 0.0408 \\
2.6 & $3.94171949 \times 10^{9}$ & $3.74229636 \times 10^{9}$ & 0.0506 \\
2.8 & $1.54972056 \times 10^{13}$ & $1.45539824 \times 10^{13}$ & 0.0609 \\
3.0 & $7.21884326 \times 10^{16}$ & $6.70106781 \times 10^{16}$ & 0.0717 \\
\hline
\end{tabular}

\section{REFERENCES}

1. L. Carlitz, On some polynomials of Tricomi, Boll. Un. Mat. Ital., 13, 58-64, 1958. MR0103303 $(21: 2078)$

2. W.M.Y. Goh and J. Wimp, On the asymptotics of the Tricomi-Carlitz polynomials and their zero distribution. I, SIAM J. Math. Anal., 25, 420-428, 1994. MR1266567 (95b:42025)

3. W.M.Y. Goh and J. Wimp, The zero distribution of the Tricomi-Carlitz polynomials. Approximation theory and applications, Comput. Math. Appl., 33, 119-127, 1997. MR.1442066 (99e:33006)

4. J.L. López and N.M. Temme, Approximation of orthogonal polynomials in terms of Hermite polynomials. Dedicated to Richard A. Askey on the occasion of his 65th birthday, Part II, Methods Appl. Anal., 6, 131-146, 1999. MR.1803886 (2001m:33013)

5. F.W.J. Olver, Asymptotics and Special Functions, Academic Press, New York, 1974. (Reprinted by A. K. Peters, Ltd., Wellesley, 1997.) MR0435697 (55:8655)

6. G. Szegö, Orthogonal Polynomials, 4th edition, Amer. Math. Soc. Colloq. Publ., 23, Amer. Math. Soc., Providence, RI, 1975. MR0372517(51:8724)

7. F.G. Tricomi, A class of non-orthogonal polynomials related to those of Laguerre, J. Analyse Math., 13, 209-231, 1951. MR0051351(14:466e)

8. Z. Wang and R. Wong, Uniform asymptotic expansion of $J_{\nu}(\nu a)$ via a difference equation, Numer. Math., 91, 147-193, 2002. MR1896091 (2003g:33008) 
9. Z. Wang and R. Wong, Asymptotic expansions for second-order linear difference equations with a turning point, Numer. Math., 94, 147-194, 2003. MR.1971216 (2004c:39012)

10. R. Wong, Asymptotic Approximations of Integrals, Academic Press, Boston, 1989. MR:1016818 (90j:41061)

Department of Mathematics, City University of Hong Kong, 83 Tat Chee Avenue, KOWLOON, Hong Kong

E-mail address: charleslkf8571@gmail.com

Department of Mathematics, City University of Hong Kong, 83 Tat Chee Avenue, Kowloon, Hong Kong

E-mail address: rscwong@cityu.edu.hk 